\title{
Seasonal variations of heavy metals concentrations in mullet, Mugil cephalus and Liza ramada (Mugilidae) from Lake Manzala , Egypt
}

\author{
Mohamed H. Bahnasawy, Abdel Aziz A. Khidr, Nadia A. Dheina \\ Zoology Department, Damietta Faculty of Science, Damietta, Egypt
}

\begin{abstract}
Seasonal variations in the concentrations of four heavy metals; zinc ( $\mathrm{Zn}$ ), $N_{\text {copper }}(\mathrm{Cu})$, lead $(\mathrm{Pb})$ and cadmium $(\mathrm{Cd})$, were determined in gills, skin and muscles of two fish species (Mugil cephalus and Liza ramada) from five locations in Lake Manzala. The average concentrations of the metals in fish tissues exhibited the following order: $\mathrm{Zn}>\mathrm{Cu}>\mathrm{Pb}>\mathrm{Cd}$. The statistical analysis revealed a significant effect of seasons, locations and fish tissues for all metals measured. The highest values of the metals were recorded in hot seasons (summer and spring). Fish samples from location V (Bahr El-Bakar) displayed the highest metal concentrations in their tissues. The highest concentrations of heavy metals were found in gills tissue of both fish species, while the lowest ones were recorded in muscles tissue. The values of the metals detected in the edible fish muscles were within the permissible limits.
\end{abstract}

Key words: Lake Manzala, heavy metals, Mugil cephalus, Liza ramada

\section{INTRODUCTION}

Fish is one of our most valuable sources of protein food. Worldwide people obtain about $25 \%$ of their animal protein from fin fish and shellfish. The protein found in fish is of high biological value, which means that fish can be used as the sole source of protein in the diet. But the real importance of fish in the diet is not its protein, but the omega-3 fat it contains. Omega- 3 fatty acids are very important for normal growth and help prevent heart disease because they make the blood less likely to form clots that cause heart attacks.

In Egypt, mullet fish especially Mugil cephalus and Liza ramada are economically very important fish because they have high market value and have been cultivated successfully by fish farmers.

Aquatic systems became contaminated with heavy metals released from domestic, industrial, mining and agricultural effluents which are continuously discharged into them (Canli et al.,1998; Shrivastava et al.,2003; Tulonen et al.,2006). Many fish species are among the top consumers of trophic pyramids in aquatic ecosystems. In consequence, they are endangered by diet-borne pollutants (e.g heavy metals) transferred along the food chain (Moriarty, 1984; Khallaf et al.,1998; Karadede et al.,2004). Because heavy metals tend to accumulate in different body organs, these metals are dangerous for fish and in 
turn they led to serious problems in both man and animals (Currey et al., 1992; Marzouk, 1994).

Fish have been used for many years to determine the pollution status of water, and are thus regarded as an excellent biological marker of metals in aquatic ecosystems (Rashed,2001; Coetzee et al.,2002; Benson et al.,2007).

Lake Manzala is considered one of the most important lakes in Egypt, that is exposed to high levels of pollutants from industrial, domestic and agricultural resources (Badawy and Wahaab, 1997; Abdel-Baky et al., 1998b; Ibrahim et al., 1999).

The main objective of this study is to evaluate heavy metals concentration in muscles, gills and skin of Mugil cephalus and Liza ramada collected from different sites in the lake during different seasons.

\section{MATERIALS AND METHODS}

Fish samples (Mugil cephalus and Liza ramada) were collected from five sites in Lake Manzala (Fig.1).

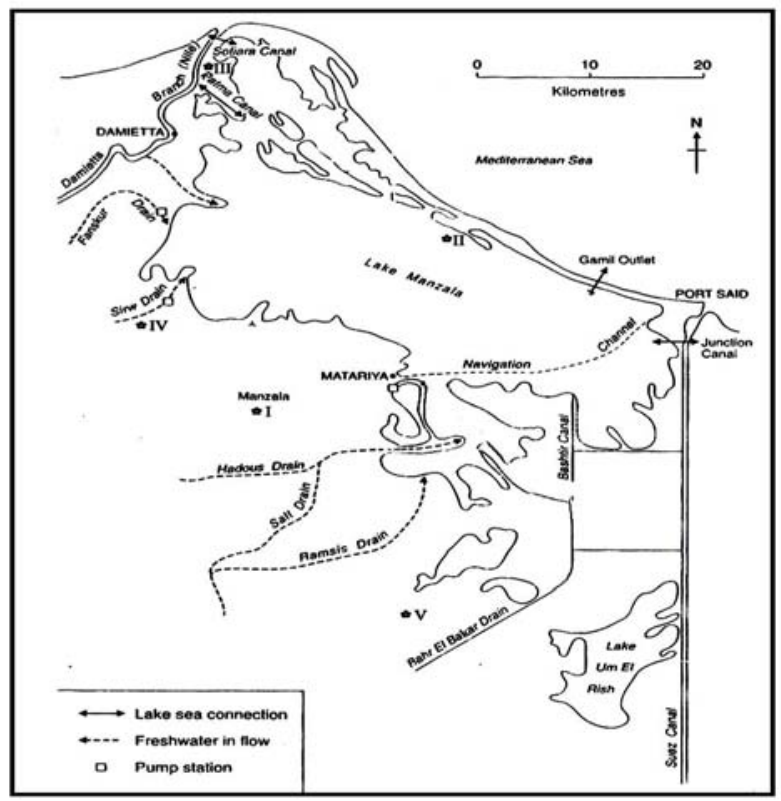

Fig. (1): Location of sampling sites $\left(^{*}\right)$ in Lake Manzala ; El-Manzala (I), El-Diba (II), El-Ratama (III), El-Sirw (IV) and Bahr El-Bakar (V)

These sites were chosen in relation to contamination gradients. Sites I\&IV receive agricultural drainage water via Hadous and El-Sirw drains. Sites II\&III are impacted partially by brackish water from the south eastern side of the lake and the saline water of Mediterranean Sea in the north. Site V receives huge amounts of sewage and industrial wastes via Bahr El-Bakar drain. The fish 
samples were placed in ice box and immediately brought to the laboratory, where they were kept deeply frozen at $-20^{\circ} \mathrm{C}$ until the samples were prepared for digestion and analysis. Before analysis, each individual fish was measured, weighed and dissected where pieces of gill, skin without scales and epaxial muscle were taken, placed in separately pre-weighed acid cleaned flasks, dried at $80^{\circ} \mathrm{C}$ using an oven and digested on a hot plate using Nitric acid and Perchloric acid (2:1). Completely digested samples were filtered through an acid-resistant filter paper and the filtrate made up to a known volume $(20 \mathrm{ml})$ with distilled water (Canli et al., 1998).

Assessment of metals $(\mathrm{Zn}, \mathrm{Cu}, \mathrm{Cd}$ and $\mathrm{Pb}$ ) levels in the prepared samples were carried out using an Atomic Absorption Spectrophotometer at Chemistry Department, Damietta Faculty of Science, Mansoura University. Statistical analysis of the obtained data was carried out using SPSS program. Two-Way ANOVA was employed to find the significant difference of heavy metals concentrations in fish organs with regard to sites and seasons. The significance level was $\mathrm{P}<0.05$ (Bailey, 1982).

\section{RESULTS}

Mean concentrations and associated standard deviations of $\mathrm{Cu}, \mathrm{Zn}, \mathrm{Cd}$ and $\mathrm{Pb}$ in gills, skin and muscle of M.cephalus and L.ramada from 5 stations in Lake Manzala are shown in tables (1-8). Figure 1 shows the sampling stations in Lake Manzala. The measured metals in the two fish species showed highly significant difference between organs, stations and seasons $(\mathrm{P}<0.05)$. Station $\mathrm{V}$ generally showed the highest heavy metal concentrations. Different tissues showed different capacities for accumulating heavy metals. The highest metal concentrations were found in gills, while the lowest levels of the metals were recorded in muscle. Metals concentrations in gills, skin and muscle of the examined fish follow the sequence: $\mathrm{Zn}>\mathrm{Cu}>\mathrm{Pb}>\mathrm{Cd}$.

\section{Metals accumulation in $M$. cephalus:}

$\mathrm{Cu}$ concentration in gills, skin and muscle ranged from 12.78 to 17.57 , from 7.59 to 10.53 and from 3.56 to $5.68 \mu \mathrm{g} / \mathrm{g}$ dry weight respectively. Summer had the highest $\mathrm{Cu}$ concentration, while the lowest was occurred during winter (Table 1).

Zn concentration ranged from 48.18 to 141.98 in gills, from 27.4 to 89.61 in skin and from 13.21 to $38.42 \mu \mathrm{g} / \mathrm{g}$ dry weight in muscle. The highest concentrations of $\mathrm{Zn}$ in the different fish organs were found during summer, while the lowest levels were recorded during winter (Table 2).

Cd concentration in gills ranged from 3.14 to $6.26 \mu \mathrm{g} / \mathrm{g}$ dry weight, while its concentration in skin ranged from 1.99 to 3.01 and in muscle from 1.08 to $1.71 \mu \mathrm{g} / \mathrm{g}$ dry weight. The highest levels of $\mathrm{Cd}$ in gills, skin and muscle were recorded during summer (Table 3 ).

$\mathrm{Pb}$ concentration in gills, skin and muscle were observed to be from 8.21 to 12.67 , from 2.32 to 3.71 and from 1.66 to $2.98 \mu \mathrm{g} / \mathrm{g}$ dry weight respectively. 
The higher value of $\mathrm{Pb}$ was recorded during summer for gills and muscle, and during spring for skin (Table 4).

\section{Metals accumulation in $L$. ramada:}

$\mathrm{Cu}$ concentration fluctuated from 8.13 to 19.97 in gills, from 5.74 to 9.55 in skin and from 3.03 to $4.66 \mu \mathrm{g} / \mathrm{g}$ dry weight in muscle. The highest levels of $\mathrm{Cu}$ were reported in summer for skin, in spring for gills and muscle (Table 5).

$\mathrm{Zn}$ concentration in gills ranged from 46.9 to 138.30 , in skin from 27.10 to 86.40 and in muscle from 12.60 to $36.90 \mu \mathrm{g} / \mathrm{g}$ dry weight. $\mathrm{Zn}$ levels were highest in summer for gills and muscle, in spring for skin (Table 6).

Cd levels ranged from 1.63 to 5.92 in gills, from 1.04 to 2.15 in skin, and from 0.51 to $1.11 \mu \mathrm{g} / \mathrm{g}$ dry weight in muscles. The highest $\mathrm{Cd}$ values were recorded in summer for gills, skin and muscle (Table 7).

$\mathrm{Pb}$ concentration varied from 5.36 to 11.52 in gills, from 2.31 to 3.31 in skin, and from 1.43 to $2.43 \mu \mathrm{g} / \mathrm{g}$ dry weight in muscle. Higher $\mathrm{Pb}$ values were recorded in summer for gills and in spring for skin and muscle (Table 8 ).

\section{DISCUSSION}

Fish samples from station $\mathrm{V}$ displayed the highest metal concentrations in their tissues. This confirms the previous findings on the same station since it receives huge amounts of sewage, industrial and agricultural wastes via Bahr ElBakar drain, which collects these pollutants from different districts through its way from Cairo (Badawy and Wahaab, 1997; Abdel-Baky et al.,1998a\&b; Ibrahim et al.,1999a; Abdel-Baky, 2001; Bahnasawy, 2001; Khalil and Faragallah, 2008 ). The fore mentioned authors demonstrated that fish surviving at highly polluted areas accumulate higher levels of heavy metals than those surviving at less polluted area of the same lake.

The phenomenon that different metals are accumulated at different concentrations in various organs and tissues of fish was observed in the present study. The difference in the levels of accumulation in different organs/tissues of a fish can primarily be attributed to the differences in the physiological role of each organ. Regulatory ability, behavior and feeding habits are other factors that influence the accumulation differences in different organs (Kotze et al., 1999). Gills of the examined fish contained the highest concentration of all the detected metals, while muscles appeared to be the least preferred site for the bioaccumulation of metals. Higher metal concentrations in the gills could be due to the element complexion with the mucus that is hardly removed from the gill lamellae before tissue analysis (Karadede et al., 2004). The adsorption of metals onto the gills surface, as the first target for pollutants in water, could also be an important influence in the total metal levels of the gill (Heath, 1987). Target organs, such as the liver and gills, are metabolically active tissues and accumulate heavy metals in higher levels, as shown in many species of fish in different areas: in $M$. cephalus in the Mediterranean Sea (Abdel-Moneim et al.,1994), in Clarias gariepinus and Labeo umbratus from Olifants River, South 
Africa (Coetzee et al.,2002), in Cyprinus carpio and Tinca tinca from Lake Beysehir, Turkey (Altindag and Yigit, 2005), in Liza abu from Ataturk Dam Lake, Turkey (Karadede et al., 2004), in Oreochromis mossambicus and Clarias gariepinus from Olifant River, South Africa (kotze et al.,1999), in Liza abu from Tigris River, Turkey (Unlu et al.,1996), in Tilapia zilli from River Nile (Hamed,1998), in M.cephalus from the northeast Mediterranean Sea, Turkey (Kalay et al.,1999; Canli and Atli, 2003). In support to this, Deb and Fukushima (1999) added that metals may be in high concentrations in the gills, intestine and digestive glands. These organs have relatively high potential for metal accumulation.

Muscles in the present study, contained the lowest levels of heavy metals. This result agrees with many authors who reported that muscle is not an active tissue in accumulating heavy metals (Unlu et al.,1996 ; Canli et al., 1998; Ibrahim et al.,1999a\&b ; Kalay et al., 1999; Canli and Atli,2003; Karadede et al., 2004 ; Yilmaz, 2005 and Chouba et al., 2007). The concentration of heavy metals in the present study, was higher in skin samples than in muscles. The reason for high metal concentration in the skin could be due to the metal complexion with the mucus that is impossible to be removed completely from the tissue before the analysis (Yilmaz, 2005). In this respect, Coetzee et al. (2002) mentioned that skin is an important excretory organ for heavy metals by means of the mucus.

In the present study, the levels of heavy metals in different fish organs showed highly significant differences between seasons. The measured metals attained their maximum values during summer, while their lowest values were found during winter. The concentration of metals in the surrounding water was also higher in summer and lower in winter (Bahnasawy et al., in press). On the other hand, Ansari et al.(2004) reported that variations of the metals concentration at a given site may be often due to seasonal changes of the organisms tissues weight rather than to any variability in the absolute metal content of the organism. The seasonal variations of heavy metals in fish were reported by many authors (Zyadah, 1997; Hamed, 1998; Khallaf et al., 1998; Ibrahim et al., 1999a\&b; Ibrahim et al., 2000).

The results of the present study showed that metals were more concentrated in $M$. cephalus tissues than those of $L$. ramada. Species differences in heavy metal bioaccumulation could be ascribed to differences in feeding habits and behaviour of the species (Kotze et al., 1999; Ibrahim et al., 1999a\&b; Mormede and Davies, 2001; Coetzee et al., 2001; Canli and Atli, 2003; Ali and Abdel-Satar, 2005; Canbek et al., 2007). Although both M.cepalus and L.ramada are pelagic fish, they differ in habitat and feeding behaviour. M.cephalus tends to live near the sediment region. Kilgour (1991) indicated that animals which have close relationship with sediment, show relatively high body concentrations of metals. 
Compared with other studies, Windom et al. (1973) found higher levels of $\mathrm{Cu}(19.0)$ and $\mathrm{Zn}(170.0) \mu \mathrm{g} / \mathrm{g}$ dry wt. in muscles of $M$. cephalus from North Atlantic. Hemens and Connell (1975) measured higher concentration of $\mathrm{Zn}$ (4261) and lower level of $\mathrm{Pb}(0.68-0.73) \mu \mathrm{g} / \mathrm{g}$ dry wt. in the muscle of Mugil spp. from the Mhlathuze Estuary, South Africa. Enormously higher values of $\mathrm{Zn}$ (210.0) and $\mathrm{Cu}(43.0) \mu \mathrm{g} / \mathrm{g}$ dry wt were found by Bebbington et al. (1977) in the muscle of M. cephalus from the coast of Australia. M. cephalus from the northern coast of Mauritania in the Atlantic ocean showed lower levels of $\mathrm{Cu}$ (2.3), $\mathrm{Cd}(<0.1)$ and $\mathrm{Pb}(<0.5)$ but a higher level of $\mathrm{Zn}(142)$ ppm dry wt. (Romeo, 1987). M. cephalus from the middle eastern coast of Tunisia showed in their muscle higher levels of $\mathrm{Cu}$ (4.78), $\mathrm{Zn}$ (45.0), but lower level of $\mathrm{Cd}(0.07)$ $\mu \mathrm{g} / \mathrm{g}$ dry wt respectively (Hamza-Chaffai et al., 1996). Muscles and gills of Liza abue from the Tigris River (Turkey) accumulated enormously higher concentrations of $\mathrm{Cu}(23.16,78.46)$ and $\mathrm{Zn}(27.26,88.74) \mu \mathrm{g} / \mathrm{g}$ dry wt respectively (Unlu et al., 1996). Abdelhamid et al. (1997) recorded higher levels of $\mathrm{Zn}(113.0-153.0), \mathrm{Pb}(13.7-15.0)$ and $\mathrm{Cd}(1.54-1.48) \mu \mathrm{g} / \mathrm{g}$ dry wt in muscles of M.cephalus from the western region of Lake Manzala . Blasco et al. (1998) measured a remarkably high concentrations of $\mathrm{Cu}$ and $\mathrm{Zn}$ in the muscle of five European Atlantic grey mullet species (L. saliens, L. aurata, L. ramada, M. cephalus and Chelon labrosus). M.cephalus from the Northeast Mediterranean Sea showed in their muscles higher concentrations of $\mathrm{Zn}$ (26.13) and $\mathrm{Pb}$ (6.25), but lower levels of $\mathrm{Cu}(4.48)$ and $\mathrm{Cd}(0.96) \mu \mathrm{g} / \mathrm{g}$ dry wt. Gills of the same fish had lower accumulations of $\mathrm{Cu}$ (7.01), $\mathrm{Zn}(43.2)$ and $\mathrm{Cd}$ (2.28) and higher level of $\mathrm{Pb}(20.84) \mu \mathrm{g} / \mathrm{g}$ dry wt. (Kalay et al. 1999). Canli and Atli (2003) recorded also higher concentrations of $\mathrm{Zn} \mathrm{(37.39)} \mathrm{and} \mathrm{Pb}$ (5.32) and lower $\mathrm{Cu}$ (4.41) and $\mathrm{Cd}(0.66) \mu \mathrm{g} / \mathrm{g}$ dry wt. in the muscle of $M$. cephalus from the North east Mediterranean Sea. The gills of this fish showed lower accumulations of $\mathrm{Cu}$ (13. 48), $\mathrm{Zn}$ (71.21), $\mathrm{Cd}$ (2.08) but higher $\mathrm{Pb}(8.95) \mu \mathrm{g} / \mathrm{g}$ dry wt. The variation of heavy metal concentrations in fish from different areas of the world may be possibly due to differences in metal concentrations and chemical characteristics of water from which fish were sampled, ecological needs, metabolism and feeding patterns of fishes and also the season in which studies were carried out (Canli et al., 1998). According to NHMRC (1987) (cited after Beldi et al., (2006)), the values of heavy metals in the muscles of M. cephalus and L. ramada were low as compared to the maximum acceptable limits. Therefore, fish muscles in the present study are considered safe for human consumption.

\section{REFERENCES}

Abdel moneim, M.; Khaled, A. and Iskander, M.(1994). A study on the levels of some heavy metals in EL-mex, west of Alexandria ,Egypt .The 4th conf. of the Environ. Prot. is a must, 10-12 May: 155-174. 
Abdel-Baky, T. E.; Hagras, A. E.; Hassan, S. H. and Zyadah, M. A. (1998a). Environment assessment of pollution in Lake Manzalz. 1- Distribution of some heavy metals in water and sediment. J. Egypt. Ger. Soc. Zool., 26 (B): 25-38.

Abdel-Baky, T. E.; Hagras, A. E.; Hassan, S. H. and Zyadah, M. A. (1998b). Heavy metals concentration in some organs of Oreochromis aureus stein in Lake Manzala, Egypt. J. Egypt. Ger. Soc. Zool., 25(A): 237-256.

Abdelhamid, A. M.; Asmaa, A.; El-Kerdawy, A. A.; El-Mezaien, A. and Meshref, H. A. (1997). Study on pollution in the Western-North region of El-Manzalah Lake, Egypt. J. Agric. Sci. Mansoura Uiv., 22 (6): 18771885 .

Abdel-moneim, M. A.; Khaled, A. M. and Iskander, M. F. (1994). A study on the levels of some heavy metals in El-Mex, West of Alexandria, Egypt. Proc. $4^{\text {th }}$ conf. of the Envir. Prot. Is a must, 10-12 May, pp: 155-174.

Ali, M. and Abdel -Satar ,A. (2005). Studies of some heavy metals in water, sediment, fish and fish diets in some fish farms in EL-Fayoum province. Egypt. J. Aquat. Res. 31(2):261-273.

Altindag, A. and Yigit, S. (2005). Assessment of heavy metal concentration in the food web of lake Beysehir, Turkey. Chemosphere .60:552-556.

Ansari, T.; Marr, I. and Tariq, N. (2004). Heavy metals in marine pollution perspective- A mini review. Journal of applied science, 4 (1): 1-20.

Badawy, M. I. and Wahaab, R. A. (1997). Environmental impact of some chemical pollutants on Lake Manzala. International Journal of Environmental Health Research, 7: 161-170.

Bahnasawy, M. (2001). Levels of heavy metals in cat fish, Clarias gariepinus, from different habitats and their effects on some biochemical parameters. Egypt. J. Aquat. Biol.\& Fish., 5(1): 99-125.

Bahnasawy,M.; Khidr,A. and Dheina,N. (in press). Assessment of heavy metals concentration in water, plankton and fish of Lake Manzala, Egypt. 
Bailey, N. (1982). Statistical methods in biology. $2^{\text {nd }}$ Ed. (Biological Science Texts), pp. 216.

Bebbington, G.; Mackay, N.; Chvojka, R.; Williams, R.; Dunn, A. and Auty, E. (1977). Heavy metals, selemium and arsenic in nine species of Australian commercial fish. Aust. J. Mar. Fresh Res. 28: 277-286.

Beldi, H.; Gimbert, F.; Maas, S.; Scheifler, R. and Soltani, N.(2006). Seasonal variations of $\mathrm{Cd}, \mathrm{Cu}, \mathrm{Pb}$ and $\mathrm{Zn}$ in the edible mollusk Donax trunculus (Mollusca, Bivalvia) from the gulf of Annaba, Algeria. Afric. J. Agric. Res.1 (4):85-90.

Benson, N.; Essien, j.; Williams, A. and Bassey, D. (2007). Mercury accumulation in fishes from Tropical aquatic ecosystem in the Niger Delta , Nigeria .Current Science . 92(6)781-785

Blasco, J.; Rubio, J. A.; Forja, J.; Gomez-parra, A. and Establier, R. (1998). Heavy metals in some fishes of the mugilidae family from salt-ponds of Cadiz Bay, Spain. Ecotoxicol. Environ. Restor., 1 ( 2 ): 71-77.

Blasco, J.; Rubio, J. A.; Forja, J.; Gomez-parra, A. and Establier, R. (1998). Heavy metals in some fishes of the Mugilidae family from salt- ponds of Cadiz Bay, Spain. Ecotoxicol. Environ. Restor., 1 ( 2 ): 71-77.

Canbek, M.; Demir, T; Uyanoglu, M; Bayram O.; Emiroglu, O.; Arslan, N. and koyuncu, O. (2007). preliminary assessment of heavy metals in water and some Cyprinidae species from the porsuk River ,Turkey. j. Appl . Biol .sci. 3: 91-95.

Canli, M. and Atli, G. (2003). The relationships between heavy metal $(\mathrm{Cd}, \mathrm{Cr}$, $\mathrm{Fe}, \mathrm{Pb}, \mathrm{Zn}$ ) levels and the size of six Mediterranean fish species. Environ. Pollut., 121:129-136.

Canli, M.; Ay, Ö. and Kalay, M. (1998). Levels of heavy metals (Cd, Pb, Cu, Cr and $\mathrm{Ni}$ ) in tissues of Cyprinus carpio, Barbus capito and Chondrostoma regium from the Seyhan River, Turkey. Turkish, J. Zoolo., 22: 149-157.

Chouba .L.; Kraiem, M.; Njimi, W.; Tissaoui, C.; Thompson, J. and Flower, R. (2007). Seasonal Variation of heavy metals $(\mathrm{Cd}, \mathrm{Pb}$, and $\mathrm{Hg}$ ) in 
sediments and mullet, Mugil cephalus ( Mugilidae) from the Ghar EL-Melh Lagoon (Tunisia). Transitional water Bulletin, 4:45-52.

Coetzee, L.; du Preez, H. H. and van Vuren, J. H. (2002). Metal concentrations in Clarias gariepinus and Labeo umbratus from the Olifant and Klein Olifants River, Mpumalanga, South Africa: Zinc, copper, manganese, lead, chromium, nickel, aluminium and iron. Water SA, 28 (4): 433-448.

Currey, N.; Benko, W.; Yaru, B. and Kabi, R. (1992). Determination of heavy metals, arsenic and selenium in barramundic (Lates calcorifer) from Lake Murray, Papua, New Guinea. Sci. Total Environ., 128:305-320.

Deb, S. and Fukushima, T. (1999). Metal in aquatic ecosystem: mechanisms of uptake, accumulation and release. Int. J. Environ. Stud., 56(3):385-433.

Hamed, M. (1998): Distribution of trace metals in the River Nile Ecosystem, Damietta branch between Mansoura city and Damietta Province. J. Egypt. Ger. Soc. Zool. 27(A):399-415.

Hamza-Chaffai, A.; Romeo, M. and El-Abed, A.(1996). Heavy metals in different fishes from the middle eastern coast of Tunisia. Bull.Environ.Contam.Toxicol, 56: 766-773.

Heath, A. (1987) Water pollution and fish physiology. Florida: CRP Press, $245 p p$.

Hemens, J. and connell, A. D. (1975). Richards Bay: Southern bay conservation area. CSIR/NIWR Progress Report No. 29 CSIR, Durban, South Africa.

Ibrahim, A.; Bahnasawy, M.; Mansy, S. and EL-Fayomy, R. (1999a). Heavy metal accumulation in water, sediment and some fishes in Lake Manzala, Egypt. J. Egypt. Ger. Soc. Zool., 29(B):43-58.

Ibrahim, A.; Bahnasawy, M.; Mansy, S. and EL-Fayomy, R. (1999b). Distribution of heavy metals in the Damietta Nile Estuary ecosystem. Egypt. J. Aquat. Biol \& Fish., 3(4) :369-397. 
Kalay, M.; Ay, O. and Canli, M. (1999). Heavy metal concentrations in fish tissues from the Northeast Mediterranean Sea. Bull. Environ. Contam. Toxicol, 63:673-681.

Karadede, H; Oymak, S. A. and Ünlü, E. (2004). Heavy metals in mullet, Liza $a b u$, and Catfish, Silurus triostegus, from the Ataturk Dam Lake (Euphrates), Turkey. Environment Internationl, 30: 183-188

Khalil, M. and Faragallah, H. (2008). The distribution of some leachable and Total heavy metals in core sediments of Manzala lagoon, Egypt. Egypt. J. Aquat. Res., 34(1):1-11.

Khallaf, E. A.; Galal, M. and Authman, M. (1998). Assessment of heavy metals pollution and their effects on Oreochromis niloticus in aquatic drainge canals. J. Egypt. Ger. Soc. Zool. 26 (B): 39-74.

Kilgour, B.(1991). Cadmium uptake from cadmium - spiked sediments by four freshwater invertebrates. Bull.Environ.Contam.Toxicol, 47:70-75.

Kotze, P.; du Preez, H.H. and van Vuren, J.H.J.(1999). Bioaccumulation of copper and zinc in Oreochromis mossambicus and Clarias gariepinus from the Olifants River, Mpumalanga, South Africa. Water S.A., 25(1): $99-110$.

Marzouk, M. (1994). Fish and environmental pollution. Vet. Med. J., 42:51-52

Moriarty, F. (1984): Persistent contaminants, compartmental models and concentration along food chains. Ecol. Bull., 36: 35-45.

Mormede, S. and Davies, I. (2001). Heavy metals concentrations in commercial deep-sea fish from the Rockall Trough. Cont. shelf Res., 12(8-10):899916.

Rashed, M. (2001). Cadmium and lead levels in fish (Tilapia nilotica) tissues as a biological indicator for lake water pollution. Environ. Monitor. Assess, 68:75-89.

Romeo, M. (1987). Trace metals in fish roe from the Mauritania coast. Mar. Pollut. Bull., 18: 507-508. 
Shrivastava, p.; Saxena, A.; Swarup, A. (2003). Heavy metal pollution in a sewage -fed lake of Bhopal, India. Lakes, Reservoirs: Research and Management, 8:1-4.

Tulonen,T., Pihlstrom,M., Arvola,L., Rask,M.(2006). Concentrations of heavy metals in food web components of small, boreal lakes. Boreal Environ. Res, 11:185-194

Ünlü, E.; Akba, O.; Sevim, S. and Gümgüm, B. (1996). Heavy metal levels in mullet, Liza abu (Heckel, 1843) (Mugilidae) from the Tigris River, Turkey. Fresenius Envir. Bull., 5: 107-112.

Windom, H.; Stickeny, R.; Smith, R.; Wite , D and Taylor, F. (1973). Arsenic, cadmium, mercury and zinc in some species of North Atlantic Finfish. Journal of Fisheries Research Board of Canada 30: 275-279.

Yilmaz, B.A. (2005). comparison of Heavy metal levels of grey Mullet (Mugil cephalus) and sea Bream (Sparus aurata) caught in Iskenderun Bay (Turkey).Turk. J. Vet. Anim. Sci. 29:257-262.

Zyadah, M. (1997). A study on level of some heavy metals in River Nile estuary-Damietta branch, Egypt. Ger. J. Soc. Zool., 23 (A): 149-60. 
Table.1: Seasonal variations of copper concentration $((\mu \mathrm{g} / \mathrm{g}$ dry weight $)$ in different organs of Mugil cephalus from Lake Manzala .

\begin{tabular}{|c|c|c|c|c|c|c|c|c|c|c|}
\hline \multirow{2}{*}{ Organ } & \multirow{2}{*}{ Site } & \multicolumn{5}{|c|}{ Seasons } & \multicolumn{4}{|c|}{ ANOVA } \\
\hline & & Winter & Spring & Summer & Autumn & Total & Factor & $\mathrm{df}$ & F value & Sig. \\
\hline \multirow{5}{*}{ Gills } & I & $\begin{array}{c}13.720 \\
\pm \\
1.252 \\
\end{array}$ & $\begin{array}{c}14.100 \\
\pm \\
0.894 \\
\end{array}$ & $\begin{array}{c}14.220 \\
\pm \\
0.559 \\
\end{array}$ & $\begin{array}{c}13.910 \\
\pm \\
1.118 \\
\end{array}$ & $\begin{array}{c}13.970 \\
\pm \\
0.939 \\
\end{array}$ & Site & 4 & 68.782 & $0.000 * * *$ \\
\hline & II & $\begin{array}{c}12.780 \\
\pm \\
0.626\end{array}$ & $\begin{array}{c}13.000 \\
\pm \\
0.716\end{array}$ & $\begin{array}{c}13.120 \\
\pm \\
0.894\end{array}$ & $\begin{array}{c}13.030 \\
\pm \\
1.207\end{array}$ & $\begin{array}{c}12.980 \\
\pm \\
0.805\end{array}$ & Season & 3 & 9.691 & $0.000^{* * *}$ \\
\hline & III & $\begin{array}{c}13.050 \\
\pm \\
0.984\end{array}$ & $\begin{array}{c}13.370 \\
\pm \\
1.006\end{array}$ & $\begin{array}{c}13.510 \\
\pm \\
1.073\end{array}$ & $\begin{array}{c}13.360 \\
\pm \\
0.425 \\
\end{array}$ & $\begin{array}{c}13.320 \\
\pm \\
0.850\end{array}$ & Organ & 2 & 3392.259 & $0.000^{* * *}$ \\
\hline & IV & $\begin{array}{c}14.290 \\
\pm \\
1.319\end{array}$ & $\begin{array}{c}14.870 \\
\pm \\
1.856\end{array}$ & $\begin{array}{c}15.050 \\
\pm \\
1.632\end{array}$ & $\begin{array}{c}14.870 \\
\pm \\
1.118 \\
\end{array}$ & $\begin{array}{c}14.770 \\
\pm \\
1.386\end{array}$ & $\begin{array}{l}\text { Site } \mathrm{x} \\
\text { Season }\end{array}$ & 12 & 0.858 & 0.591 \\
\hline & V & $\begin{array}{c}15.010 \\
\pm \\
1.453\end{array}$ & $\begin{array}{c}15.620 \\
\pm \\
0.939\end{array}$ & $\begin{array}{c}17.570 \\
\pm \\
1.655\end{array}$ & $\begin{array}{c}15.640 \\
\pm \\
0.738\end{array}$ & $\begin{array}{c}15.960 \\
\pm \\
1.476\end{array}$ & $\begin{array}{l}\text { Site } \mathrm{x} \\
\text { Organ }\end{array}$ & 8 & 4.028 & $0.000^{* * *}$ \\
\hline \multirow{5}{*}{ Skin } & I & $\begin{array}{c}8.460 \\
\pm \\
0.425\end{array}$ & $\begin{array}{c}8.740 \\
\pm \\
0.581\end{array}$ & $\begin{array}{c}9.060 \\
\pm \\
0.716 \\
\end{array}$ & $\begin{array}{c}8.920 \\
\pm \\
0.716 \\
\end{array}$ & $\begin{array}{c}8.800 \\
\pm \\
0.626\end{array}$ & $\begin{array}{c}\text { Season } \mathrm{x} \\
\text { Organ }\end{array}$ & 6 & 0.392 & 0.884 \\
\hline & II & $\begin{array}{c}7.590 \\
\pm \\
0.664 \\
\end{array}$ & $\begin{array}{c}7.790 \\
\pm \\
0.559 \\
\end{array}$ & $\begin{array}{c}7.880 \\
\pm \\
0.440 \\
\end{array}$ & $\begin{array}{c}7.640 \\
\pm \\
0.427 \\
\end{array}$ & $\begin{array}{c}7.730 \\
\pm \\
0.492 \\
\end{array}$ & & & & \\
\hline & III & $\begin{array}{c}7.980 \\
\pm \\
0.872\end{array}$ & $\begin{array}{c}8.240 \\
\pm \\
0.648\end{array}$ & $\begin{array}{c}8.320 \\
\pm \\
0.470\end{array}$ & $\begin{array}{c}8.190 \\
\pm \\
0.648\end{array}$ & $\begin{array}{c}8.180 \\
\pm \\
0.621\end{array}$ & Site & 24 & 0.354 & 0.998 \\
\hline & IV & $\begin{array}{c}8.940 \\
\pm \\
0.783\end{array}$ & $\begin{array}{c}9.340 \\
\pm \\
0.447\end{array}$ & $\begin{array}{c}9.460 \\
\pm \\
0.514\end{array}$ & $\begin{array}{c}9.320 \\
\pm \\
0.537\end{array}$ & $\begin{array}{c}9.270 \\
\pm \\
0.531\end{array}$ & $\mathrm{x}$ & & & \\
\hline & V & $\begin{array}{c}9.700 \\
\pm \\
0.457\end{array}$ & $\begin{array}{c}10.280 \\
\pm \\
0.380\end{array}$ & $\begin{array}{c}10.530 \\
\pm \\
0.939\end{array}$ & $\begin{array}{c}10.330 \\
\pm \\
0.827\end{array}$ & $\begin{array}{c}10.210 \\
\pm \\
0.716\end{array}$ & Season & & & \\
\hline \multirow{5}{*}{ Muscles } & I & $\begin{array}{c}4.190 \\
\pm \\
0.521\end{array}$ & $\begin{array}{c}4.760 \\
\pm \\
0.693\end{array}$ & $\begin{array}{c}4.850 \\
\pm \\
0.335 \\
\end{array}$ & $\begin{array}{c}4.640 \\
\pm \\
0.760 \\
\end{array}$ & $\begin{array}{c}4.610 \\
\pm \\
0.620\end{array}$ & $\mathrm{x}$ & & & \\
\hline & II & $\begin{array}{c}3.560 \\
\pm \\
0.604\end{array}$ & $\begin{array}{c}3.930 \\
\pm \\
0.470\end{array}$ & $\begin{array}{c}4.120 \\
\pm \\
0.648 \\
\end{array}$ & $\begin{array}{c}3.890 \\
\pm \\
0.358 \\
\end{array}$ & $\begin{array}{c}3.870 \\
\pm \\
0.537 \\
\end{array}$ & Organ & & & \\
\hline & III & $\begin{array}{c}4.170 \\
\pm \\
0.514 \\
\end{array}$ & $\begin{array}{c}4.380 \\
\pm \\
0.805 \\
\end{array}$ & $\begin{array}{c}4.580 \\
\pm \\
0.671 \\
\end{array}$ & $\begin{array}{c}4.410 \\
\pm \\
0.447 \\
\end{array}$ & $\begin{array}{c}4.390 \\
\pm \\
0.581 \\
\end{array}$ & & & & \\
\hline & IV & $\begin{array}{c}4.340 \\
\pm \\
0.827\end{array}$ & $\begin{array}{c}5.130 \\
\pm \\
0.588\end{array}$ & $\begin{array}{c}5.210 \\
\pm \\
0.984\end{array}$ & $\begin{array}{c}4.970 \\
\pm \\
0.612\end{array}$ & $\begin{array}{c}4.910 \\
\pm \\
0.805\end{array}$ & & & & \\
\hline & V & $\begin{array}{c}4.530 \\
\pm \\
0.738\end{array}$ & $\begin{array}{c}5.300 \\
\pm \\
0.581\end{array}$ & $\begin{array}{c}5.680 \\
\pm \\
0.559\end{array}$ & $\begin{array}{c}5.350 \\
\pm \\
0.462\end{array}$ & $\begin{array}{c}5.210 \\
\pm \\
0.716\end{array}$ & & & & \\
\hline
\end{tabular}


Table.2: Seasonal variations of Zinc concentration ( $\mu \mathrm{g} / \mathrm{g}$ dry weight) in different organs of Mugil

\begin{tabular}{|c|c|c|c|c|c|c|c|c|c|c|}
\hline \multirow{2}{*}{ Organ } & \multirow{2}{*}{ Site } & \multicolumn{5}{|c|}{ Seasons } & \multicolumn{4}{|c|}{ ANOVA } \\
\hline & & Winter & Spring & Summer & Autumn & Total & Factor & df & F value & Sig. \\
\hline \multirow{5}{*}{ Gills } & I & $\begin{array}{c}51.270 \\
\pm \\
20.326\end{array}$ & $\begin{array}{c}87.780 \\
\pm \\
25.335 \\
\end{array}$ & $\begin{array}{c}89.770 \\
\pm \\
9.973 \\
\end{array}$ & $\begin{array}{c}56.320 \\
\pm \\
22.741 \\
\end{array}$ & $\begin{array}{c}71.290 \\
\pm \\
26.028 \\
\end{array}$ & Site & 4 & 55.012 & $0.000 * * *$ \\
\hline & II & $\begin{array}{c}49.280 \\
\pm \\
20.684\end{array}$ & $\begin{array}{c}52.320 \\
\pm \\
8.877\end{array}$ & $\begin{array}{c}72.310 \\
\pm \\
18.045\end{array}$ & $\begin{array}{c}51.020 \\
\pm \\
13.327\end{array}$ & $\begin{array}{c}56.230 \\
\pm \\
17.441\end{array}$ & Season & 3 & 83.095 & $0.000 * * *$ \\
\hline & III & $\begin{array}{c}48.180 \\
\pm \\
28.733\end{array}$ & $\begin{array}{c}59.160 \\
\pm \\
13.372\end{array}$ & $\begin{array}{c}83.720 \\
\pm \\
20.326\end{array}$ & $\begin{array}{c}66.860 \\
\pm \\
15.429 \\
\end{array}$ & $\begin{array}{c}64.480 \\
\pm \\
22.897 \\
\end{array}$ & Organ & 2 & 443.004 & $0.000 * * *$ \\
\hline & IV & $\begin{array}{c}61.240 \\
\pm \\
17.307\end{array}$ & $\begin{array}{c}92.450 \\
\pm \\
6.820\end{array}$ & $\begin{array}{c}102.990 \\
\pm \\
17.710\end{array}$ & $\begin{array}{c}76.880 \\
\pm \\
19.051\end{array}$ & $\begin{array}{c}83.390 \\
\pm \\
21.869\end{array}$ & $\begin{array}{l}\text { Site } \mathrm{x} \\
\text { Season }\end{array}$ & 12 & 2.743 & $0.002 * *$ \\
\hline & V & $\begin{array}{c}67.030 \\
\pm \\
9.839\end{array}$ & $\begin{array}{c}115.330 \\
\pm \\
29.158\end{array}$ & $\begin{array}{c}141.980 \\
\pm \\
10.219\end{array}$ & $\begin{array}{c}99.500 \\
\pm \\
19.856\end{array}$ & $\begin{array}{c}105.960 \\
\pm \\
32.825\end{array}$ & $\begin{array}{l}\text { Site } \mathrm{x} \\
\text { Organ }\end{array}$ & 8 & 6.037 & $0.000 * * *$ \\
\hline \multirow{5}{*}{ Skin } & I & $\begin{array}{c}31.410 \\
\pm \\
6.485 \\
\end{array}$ & $\begin{array}{c}53.610 \\
\pm \\
9.503 \\
\end{array}$ & $\begin{array}{c}62.660 \\
\pm \\
21.735 \\
\end{array}$ & $\begin{array}{c}33.770 \\
\pm \\
6.954 \\
\end{array}$ & $\begin{array}{c}45.360 \\
\pm \\
17.933 \\
\end{array}$ & $\begin{array}{c}\text { Season } \mathrm{x} \\
\text { Organ }\end{array}$ & 6 & 11.231 & $0.000 * * *$ \\
\hline & II & $\begin{array}{c}27.400 \\
\pm \\
3.779\end{array}$ & $\begin{array}{c}36.670 \\
\pm \\
8.475\end{array}$ & $\begin{array}{c}54.890 \\
\pm \\
11.874\end{array}$ & $\begin{array}{c}29.420 \\
\pm \\
5.188\end{array}$ & $\begin{array}{c}\begin{array}{c}37.100 \\
\pm \\
13.327\end{array} \\
\end{array}$ & & & & \\
\hline & III & $\begin{array}{c}30.480 \\
\pm \\
6.350\end{array}$ & $\begin{array}{c}52.200 \\
\pm \\
10.957\end{array}$ & $\begin{array}{c}68.200 \\
\pm \\
10.800\end{array}$ & $\begin{array}{c}31.070 \\
\pm \\
9.011\end{array}$ & $\begin{array}{c}45.490 \\
\pm \\
18.380\end{array}$ & Site & 24 & 0.954 & 0.528 \\
\hline & IV & $\begin{array}{c}32.940 \\
\pm \\
7.960\end{array}$ & $\begin{array}{c}61.570 \\
\pm \\
6.977\end{array}$ & $\begin{array}{c}80.620 \\
\pm \\
13.059\end{array}$ & $\begin{array}{c}41.480 \\
\pm \\
11.806\end{array}$ & $\begin{array}{c}54.150 \\
\pm \\
21.153\end{array}$ & $\mathrm{x}$ & & & \\
\hline & V & $\begin{array}{c}35.930 \\
\pm \\
4.517 \\
\end{array}$ & $\begin{array}{c}77.310 \\
\pm \\
14.870 \\
\end{array}$ & $\begin{array}{c}89.610 \\
\pm \\
4.673 \\
\end{array}$ & $\begin{array}{c}56.830 \\
\pm \\
6.954 \\
\end{array}$ & $\begin{array}{c}64.920 \\
\pm \\
22.450 \\
\end{array}$ & Season & & & \\
\hline \multirow{5}{*}{ Muscles } & I & $\begin{array}{c}17.720 \\
\pm \\
7.021\end{array}$ & $\begin{array}{c}23.470 \\
\pm \\
6.082 \\
\end{array}$ & $\begin{array}{c}26.680 \\
\pm \\
7.491 \\
\end{array}$ & $\begin{array}{c}21.080 \\
\pm \\
3.913\end{array}$ & $\begin{array}{c}22.240 \\
\pm \\
6.663\end{array}$ & $\mathrm{x}$ & & & \\
\hline & II & $\begin{array}{c}13.210 \\
\pm \\
2.124\end{array}$ & $\begin{array}{c}16.940 \\
\pm \\
3.712\end{array}$ & $\begin{array}{c}20.320 \\
\pm \\
4.092 \\
\end{array}$ & $\begin{array}{c}15.310 \\
\pm \\
4.271\end{array}$ & $\begin{array}{c}16.440 \\
\pm \\
4.293\end{array}$ & Organ & & & \\
\hline & III & $\begin{array}{c}17.260 \\
\pm \\
2.728\end{array}$ & $\begin{array}{c}20.620 \\
\pm \\
4.047\end{array}$ & $\begin{array}{c}22.950 \\
\pm \\
4.852 \\
\end{array}$ & $\begin{array}{c}20.570 \\
\pm \\
4.114\end{array}$ & $\begin{array}{c}20.340 \\
\pm \\
4.249\end{array}$ & & & & \\
\hline & IV & $\begin{array}{c}23.390 \\
\pm \\
2.817 \\
\end{array}$ & $\begin{array}{c}29.250 \\
\pm \\
6.015 \\
\end{array}$ & $\begin{array}{c}32.020 \\
\pm \\
5.322 \\
\end{array}$ & $\begin{array}{c}25.120 \\
\pm \\
1.744 \\
\end{array}$ & $\begin{array}{c}27.450 \\
\pm \\
5.277 \\
\end{array}$ & & & & \\
\hline & V & $\begin{array}{c}25.530 \\
\pm \\
3.175 \\
\end{array}$ & $\begin{array}{c}34.550 \\
\pm \\
9.168 \\
\end{array}$ & $\begin{array}{c}38.420 \\
\pm \\
9.118 \\
\end{array}$ & $\begin{array}{c}29.540 \\
\pm \\
5.255 \\
\end{array}$ & $\begin{array}{c}32.010 \\
\pm \\
8.273 \\
\end{array}$ & & & & \\
\hline
\end{tabular}


Table.3: Seasonal variations of cadmium concentration $(\mu \mathrm{g} / \mathrm{g}$ dry weight) in different organs of Mugil cephalus from Lake Manzala .

\begin{tabular}{|c|c|c|c|c|c|c|c|c|c|c|}
\hline \multirow{2}{*}{ Organ } & \multirow{2}{*}{ Site } & \multicolumn{5}{|c|}{ Seasons } & \multicolumn{4}{|c|}{ ANOVA } \\
\hline & & Winter & Spring & Summer & Autumn & Total & Factor & $\mathrm{df}$ & F value & Sig. \\
\hline \multirow{5}{*}{ Gills } & I & $\begin{array}{c}3.470 \\
\pm \\
1.006\end{array}$ & $\begin{array}{c}4.180 \\
\pm \\
1.118\end{array}$ & $\begin{array}{c}4.240 \\
\pm \\
1.163\end{array}$ & $\begin{array}{c}3.730 \\
\pm \\
0.671\end{array}$ & $\begin{array}{c}3.900 \\
\pm \\
0.984\end{array}$ & Site & 4 & 29.521 & $\begin{array}{l}0.000 * \\
* *\end{array}$ \\
\hline & II & $\begin{array}{c}3.140 \\
\pm \\
0.738\end{array}$ & $\begin{array}{c}3.320 \\
\pm \\
0.514\end{array}$ & $\begin{array}{c}3.620 \\
\pm \\
0.693\end{array}$ & $\begin{array}{c}3.410 \\
\pm \\
0.537\end{array}$ & $\begin{array}{c}3.370 \\
\pm \\
0.526\end{array}$ & Season & 3 & 7.685 & $\begin{array}{l}0.000 * \\
* *\end{array}$ \\
\hline & III & $\begin{array}{c}3.460 \\
\pm \\
0.470\end{array}$ & $\begin{array}{c}4.120 \\
\pm \\
0.211 \\
\end{array}$ & $\begin{array}{c}4.230 \\
\pm \\
0.931 \\
\end{array}$ & $\begin{array}{c}4.100 \\
\pm \\
1.275 \\
\end{array}$ & $\begin{array}{c}3.980 \\
\pm \\
0.850 \\
\end{array}$ & Organ & 2 & 605.169 & $\begin{array}{l}0.000 * \\
* *\end{array}$ \\
\hline & IV & $\begin{array}{c}4.840 \\
\pm \\
1.699\end{array}$ & $\begin{array}{c}5.120 \\
\pm \\
0.648\end{array}$ & $\begin{array}{c}5.610 \\
\pm \\
0.962 \\
\end{array}$ & $\begin{array}{c}5.390 \\
\pm \\
0.626 \\
\end{array}$ & $\begin{array}{c}5.240 \\
\pm \\
1.029\end{array}$ & $\begin{array}{l}\text { Site } \mathrm{x} \\
\text { Season }\end{array}$ & 12 & 0.143 & 1 \\
\hline & $\mathrm{V}$ & $\begin{array}{c}5.340 \\
\pm \\
1.766\end{array}$ & $\begin{array}{c}5.620 \\
\pm \\
1.342\end{array}$ & $\begin{array}{c}6.260 \\
\pm \\
1.252\end{array}$ & $\begin{array}{c}5.930 \\
\pm \\
1.207\end{array}$ & $\begin{array}{c}5.790 \\
\pm \\
1.342\end{array}$ & $\begin{array}{l}\text { Site } \mathrm{x} \\
\text { Organ }\end{array}$ & 8 & 12.051 & $\begin{array}{l}0.000^{*} \\
* *\end{array}$ \\
\hline \multirow{5}{*}{ Skin } & I & $\begin{array}{c}2.040 \\
\pm \\
0.176\end{array}$ & $\begin{array}{c}2.260 \\
\pm \\
0.268\end{array}$ & $\begin{array}{c}2.530 \\
\pm \\
0.124\end{array}$ & $\begin{array}{c}2.410 \\
\pm \\
0.268\end{array}$ & $\begin{array}{c}2.310 \\
\pm \\
0.268\end{array}$ & $\begin{array}{c}\text { Season } \mathrm{x} \\
\text { Organ }\end{array}$ & 6 & 0.661 & 0.681 \\
\hline & II & $\begin{array}{c}1.990 \\
\pm \\
0.112\end{array}$ & $\begin{array}{c}2.130 \\
\pm \\
0.180\end{array}$ & $\begin{array}{c}2.320 \\
\pm \\
0.169\end{array}$ & $\begin{array}{c}2.250 \\
\pm \\
0.124\end{array}$ & $\begin{array}{c}2.170 \\
\pm \\
0.171\end{array}$ & & & & \\
\hline & III & $\begin{array}{c}2.010 \\
\pm \\
0.157\end{array}$ & $\begin{array}{c}2.380 \\
\pm \\
0.086 \\
\end{array}$ & $\begin{array}{c}2.500 \\
\pm \\
0.189 \\
\end{array}$ & $\begin{array}{c}2.440 \\
\pm \\
0.201 \\
\end{array}$ & $\begin{array}{c}2.340 \\
\pm \\
0.224 \\
\end{array}$ & Site & 24 & 0.131 & 1 \\
\hline & IV & $\begin{array}{c}2.090 \\
\pm \\
0.402\end{array}$ & $\begin{array}{c}2.350 \\
\pm \\
0.492\end{array}$ & $\begin{array}{c}2.680 \\
\pm \\
0.581 \\
\end{array}$ & $\begin{array}{c}2.480 \\
\pm \\
0.380 \\
\end{array}$ & $\begin{array}{c}2.400 \\
\pm \\
0.492 \\
\end{array}$ & $\mathrm{x}$ & & & \\
\hline & V & $\begin{array}{c}2.460 \\
\pm \\
0.604\end{array}$ & $\begin{array}{c}2.740 \\
\pm \\
0.380\end{array}$ & $\begin{array}{c}3.010 \\
\pm \\
0.268\end{array}$ & $\begin{array}{c}2.640 \\
\pm \\
0.358\end{array}$ & $\begin{array}{c}2.710 \\
\pm \\
0.447\end{array}$ & Season & & & \\
\hline \multirow{5}{*}{ Muscles } & I & $\begin{array}{c}1.080 \\
\pm \\
0.134\end{array}$ & $\begin{array}{c}1.270 \\
\pm \\
0.067\end{array}$ & $\begin{array}{c}1.500 \\
\pm \\
0.291\end{array}$ & $\begin{array}{c}1.430 \\
\pm \\
0.216\end{array}$ & $\begin{array}{c}1.320 \\
\pm \\
0.268 \\
\end{array}$ & $\mathrm{x}$ & & & \\
\hline & II & $\begin{array}{c}1.180 \\
\pm \\
0.189\end{array}$ & $\begin{array}{c}1.230 \\
\pm \\
0.119\end{array}$ & $\begin{array}{c}1.330 \\
\pm \\
0.240\end{array}$ & $\begin{array}{c}1.280 \\
\pm \\
0.130\end{array}$ & $\begin{array}{c}1.260 \\
\pm \\
0.179\end{array}$ & Organ & & & \\
\hline & III & $\begin{array}{c}1.230 \\
\pm \\
0.157\end{array}$ & $\begin{array}{c}1.260 \\
\pm \\
0.201 \\
\end{array}$ & $\begin{array}{c}1.390 \\
\pm \\
0.335 \\
\end{array}$ & $\begin{array}{c}1.410 \\
\pm \\
0.179 \\
\end{array}$ & $\begin{array}{c}1.320 \\
\pm \\
0.221 \\
\end{array}$ & & & & \\
\hline & IV & $\begin{array}{c}1.310 \\
\pm \\
0.107\end{array}$ & $\begin{array}{c}1.410 \\
\pm \\
0.137\end{array}$ & $\begin{array}{c}1.540 \\
\pm \\
0.216\end{array}$ & $\begin{array}{c}1.470 \\
\pm \\
0.246\end{array}$ & $\begin{array}{c}1.430 \\
\pm \\
0.224\end{array}$ & & & & \\
\hline & V & $\begin{array}{c}1.480 \\
\pm \\
0.648\end{array}$ & $\begin{array}{c}1.670 \\
\pm \\
0.241\end{array}$ & $\begin{array}{c}1.710 \\
\pm \\
0.248\end{array}$ & $\begin{array}{c}1.640 \\
\pm \\
0.271\end{array}$ & $\begin{array}{c}1.620 \\
\pm \\
0.402\end{array}$ & & & & \\
\hline
\end{tabular}


Table.4: Seasonal variations of lead concentration ( $\mu \mathrm{g} / \mathrm{g}$ dry weight) in different organs of Mugil cephalus from Lake Manzala .

\begin{tabular}{|c|c|c|c|c|c|c|c|c|c|c|}
\hline \multirow{2}{*}{ Organ } & \multirow{2}{*}{ Site } & \multicolumn{5}{|c|}{ Seasons } & \multicolumn{4}{|c|}{ ANOVA } \\
\hline & & Winter & Spring & Summer & Autumn & Total & Factor & $\mathrm{df}$ & F value & Sig. \\
\hline \multirow{5}{*}{ Gills } & I & $\begin{array}{c}9.200 \\
\pm \\
0.827\end{array}$ & $\begin{array}{c}9.580 \\
\pm \\
1.140\end{array}$ & $\begin{array}{c}10.170 \\
\pm \\
0.738\end{array}$ & $\begin{array}{c}9.880 \\
\pm \\
0.783 \\
\end{array}$ & $\begin{array}{c}9.710 \\
\pm \\
0.864\end{array}$ & Site & 4 & 71.471 & ** $0.000^{*}$ \\
\hline & II & $\begin{array}{c}8.210 \\
\pm \\
0.604 \\
\end{array}$ & $\begin{array}{c}8.700 \\
\pm \\
1.111 \\
\end{array}$ & $\begin{array}{c}9.250 \\
\pm \\
0.850\end{array}$ & $\begin{array}{c}9.180 \\
\pm \\
1.096 \\
\end{array}$ & $\begin{array}{c}8.840 \\
\pm \\
0.984\end{array}$ & Season & 3 & 10.701 & $*^{0.000 *}$ \\
\hline & III & $\begin{array}{c}9.090 \\
\pm \\
0.201\end{array}$ & $\begin{array}{c}9.510 \\
\pm \\
0.917 \\
\end{array}$ & $\begin{array}{c}9.900 \\
\pm \\
1.118\end{array}$ & $\begin{array}{c}9.670 \\
\pm \\
0.514 \\
\end{array}$ & $\begin{array}{c}9.540 \\
\pm \\
0.760\end{array}$ & Organ & 2 & 4838.837 & ${ }_{* *}^{0.000 *}$ \\
\hline & IV & $\begin{array}{c}9.820 \\
\pm \\
0.671\end{array}$ & $\begin{array}{c}10.120 \\
\pm \\
1.275\end{array}$ & $\begin{array}{c}10.460 \\
\pm \\
1.185\end{array}$ & $\begin{array}{c}10.220 \\
\pm \\
1.140\end{array}$ & $\begin{array}{c}10.160 \\
\pm \\
1.029\end{array}$ & $\begin{array}{c}\text { Site } \mathrm{x} \\
\text { Season }\end{array}$ & 12 & 0.250 & 0.995 \\
\hline & $\mathrm{V}$ & $\begin{array}{c}11.470 \\
\pm \\
0.894\end{array}$ & $\begin{array}{c}12.480 \\
\pm \\
0.738\end{array}$ & $\begin{array}{c}12.670 \\
\pm \\
1.140\end{array}$ & $\begin{array}{c}12.300 \\
\pm \\
0.648\end{array}$ & $\begin{array}{c}12.230 \\
\pm \\
0.939\end{array}$ & $\begin{array}{c}\text { Site } \mathrm{x} \\
\text { Organ }\end{array}$ & 8 & 14.252 & $*^{0.000 *}$ \\
\hline \multirow{5}{*}{ Skin } & I & $\begin{array}{c}2.540 \\
\pm \\
0.446\end{array}$ & $\begin{array}{c}2.880 \\
\pm \\
0.693\end{array}$ & $\begin{array}{c}3.060 \\
\pm \\
0.537\end{array}$ & $\begin{array}{c}2.880 \\
\pm \\
0.268\end{array}$ & $\begin{array}{c}2.840 \\
\pm \\
0.537\end{array}$ & $\begin{array}{c}\text { Season } \mathrm{x} \\
\text { Organ }\end{array}$ & 6 & 1.275 & 0.269 \\
\hline & II & $\begin{array}{c}2.320 \\
\pm \\
0.436\end{array}$ & $\begin{array}{c}2.550 \\
\pm \\
0.517\end{array}$ & $\begin{array}{c}2.960 \\
\pm \\
0.442\end{array}$ & $\begin{array}{c}2.720 \\
\pm \\
0.243\end{array}$ & $\begin{array}{c}2.640 \\
\pm \\
0.472\end{array}$ & & & & \\
\hline & III & $\begin{array}{c}2.530 \\
\pm \\
0.335 \\
\end{array}$ & $\begin{array}{c}2.720 \\
\pm \\
0.537 \\
\end{array}$ & $\begin{array}{c}3.170 \\
\pm \\
0.470\end{array}$ & $\begin{array}{c}2.950 \\
\pm \\
0.335\end{array}$ & $\begin{array}{c}2.840 \\
\pm \\
0.447\end{array}$ & Site & 24 & 0.232 & 1 \\
\hline & IV & $\begin{array}{c}2.810 \\
\pm \\
0.246\end{array}$ & $\begin{array}{c}3.080 \\
\pm \\
0.380\end{array}$ & $\begin{array}{c}3.290 \\
\pm \\
0.693\end{array}$ & $\begin{array}{c}3.060 \\
\pm \\
0.492\end{array}$ & $\begin{array}{c}3.060 \\
\pm \\
0.452\end{array}$ & $\mathrm{x}$ & & & \\
\hline & $\mathrm{V}$ & $\begin{array}{c}3.680 \\
\pm \\
0.470\end{array}$ & $\begin{array}{c}3.710 \\
\pm \\
0.447\end{array}$ & $\begin{array}{c}3.410 \\
\pm \\
0.415\end{array}$ & $\begin{array}{c}3.700 \\
\pm \\
0.318 \\
\end{array}$ & $\begin{array}{c}3.630 \\
\pm \\
0.402\end{array}$ & Season & & & \\
\hline \multirow{5}{*}{ Muscles } & I & $\begin{array}{c}1.940 \\
\pm \\
0.221\end{array}$ & $\begin{array}{c}2.110 \\
\pm \\
0.268\end{array}$ & $\begin{array}{c}2.280 \\
\pm \\
0.537\end{array}$ & $\begin{array}{c}2.200 \\
\pm \\
0.224\end{array}$ & $\begin{array}{c}2.130 \\
\pm \\
0.322\end{array}$ & $\mathrm{x}$ & & & \\
\hline & II & $\begin{array}{c}1.660 \\
\pm \\
0.112 \\
\end{array}$ & $\begin{array}{c}1.810 \\
\pm \\
0.470 \\
\end{array}$ & $\begin{array}{c}1.980 \\
\pm \\
0.224 \\
\end{array}$ & $\begin{array}{c}1.860 \\
\pm \\
0.402 \\
\end{array}$ & $\begin{array}{c}1.830 \\
\pm \\
0.313\end{array}$ & Organ & & & \\
\hline & III & $\begin{array}{c}1.680 \\
\pm \\
0.173 \\
\end{array}$ & $\begin{array}{c}1.770 \\
\pm \\
0.358 \\
\end{array}$ & $\begin{array}{c}\begin{array}{c}2.100 \\
\pm \\
0.305\end{array} \\
\end{array}$ & $\begin{array}{c}1.880 \\
\pm \\
0.134 \\
\end{array}$ & $\begin{array}{c}1.860 \\
\pm \\
0.310\end{array}$ & & & & \\
\hline & IV & $\begin{array}{c}2.240 \\
\pm \\
0.355\end{array}$ & $\begin{array}{c}2.460 \\
\pm \\
0.169\end{array}$ & $\begin{array}{c}2.530 \\
\pm \\
0.559\end{array}$ & $\begin{array}{c}2.260 \\
\pm \\
0.447\end{array}$ & $\begin{array}{c}2.370 \\
\pm \\
0.422\end{array}$ & & & & \\
\hline & V & $\begin{array}{c}2.540 \\
\pm \\
0.204 \\
\end{array}$ & $\begin{array}{c}2.870 \\
\pm \\
0.268 \\
\end{array}$ & $\begin{array}{c}2.980 \\
\pm \\
0.425\end{array}$ & $\begin{array}{c}2.760 \\
\pm \\
0.429\end{array}$ & $\begin{array}{c}2.790 \\
\pm \\
0.358\end{array}$ & & & & \\
\hline
\end{tabular}


Table.5: Seasonal variations of copper concentration $((\mu \mathrm{g} / \mathrm{g}$ dry weight $)$ in different organs of Liza ramada from Lake Manzala .

\begin{tabular}{|c|c|c|c|c|c|c|c|c|c|c|}
\hline \multirow{2}{*}{ Organ } & \multirow{2}{*}{ Site } & \multicolumn{5}{|c|}{ Seasons } & \multicolumn{4}{|c|}{ ANOVA } \\
\hline & & Winter & Spring & Summer & Autumn & Total & Factor & df & F value & Sig. \\
\hline \multirow{5}{*}{ Gills } & I & $\begin{array}{c}11.462 \\
\pm \\
1.559\end{array}$ & $\begin{array}{c}13.130 \\
\pm \\
2.859\end{array}$ & $\begin{array}{c}13.530 \\
\pm \\
5.299\end{array}$ & $\begin{array}{c}12.522 \\
\pm \\
3.104\end{array}$ & $\begin{array}{c}12.661 \\
\pm \\
3.288\end{array}$ & Site & 4 & $\begin{array}{c}10.65 \\
1\end{array}$ & $\begin{array}{l}0.000^{*} \\
* *\end{array}$ \\
\hline & II & $\begin{array}{c}10.354 \\
\pm \\
1.588\end{array}$ & $\begin{array}{c}11.368 \\
\pm \\
3.590\end{array}$ & $\begin{array}{c}14.866 \\
\pm \\
2.905\end{array}$ & $\begin{array}{c}11.914 \\
\pm \\
2.234\end{array}$ & $\begin{array}{c}11.569 \\
\pm \\
3.477\end{array}$ & Season & 3 & 9.674 & $\begin{array}{l}0.000^{*} \\
* *\end{array}$ \\
\hline & III & $\begin{array}{c}8.130 \\
\pm \\
0.645\end{array}$ & $\begin{array}{c}12.696 \\
\pm \\
2.380\end{array}$ & $\begin{array}{c}13.000 \\
\pm \\
4.915\end{array}$ & $\begin{array}{c}12.490 \\
\pm \\
0.322\end{array}$ & $\begin{array}{c}12.135 \\
\pm \\
2.745\end{array}$ & Organ & 2 & $\begin{array}{l}444.3 \\
35\end{array}$ & $\begin{array}{l}0.000 * \\
* *\end{array}$ \\
\hline & IV & $\begin{array}{c}11.544 \\
\pm \\
3.349\end{array}$ & $\begin{array}{c}14.852 \\
\pm \\
6.112\end{array}$ & $\begin{array}{c}14.460 \\
\pm \\
5.638\end{array}$ & $\begin{array}{c}13.848 \\
\pm \\
2.684\end{array}$ & $\begin{array}{c}13.676 \\
\pm \\
4.491 \\
\end{array}$ & $\begin{array}{l}\text { Site } \mathrm{x} \\
\text { Season }\end{array}$ & 12 & 0.264 & 0.994 \\
\hline & V & $\begin{array}{c}14.368 \\
\pm \\
4.817\end{array}$ & $\begin{array}{c}16.970 \\
\pm \\
3.195\end{array}$ & $\begin{array}{c}15.430 \\
\pm \\
4.402\end{array}$ & $\begin{array}{c}14.890 \\
\pm \\
3.785\end{array}$ & $\begin{array}{c}15.416 \\
\pm \\
3.889\end{array}$ & $\begin{array}{l}\text { Site } \mathrm{x} \\
\text { Organ }\end{array}$ & 8 & 1.406 & 0.194 \\
\hline \multirow{5}{*}{ Skin } & I & $\begin{array}{c}7.468 \\
\pm \\
1.629\end{array}$ & $\begin{array}{c}7.884 \\
\pm \\
0.427\end{array}$ & $\begin{array}{c}8.390 \\
\pm \\
0.141\end{array}$ & $\begin{array}{c}7.234 \\
\pm \\
0.083\end{array}$ & $\begin{array}{c}7.744 \\
\pm \\
0.898\end{array}$ & $\begin{array}{c}\text { Season } \mathrm{x} \\
\text { Organ }\end{array}$ & 6 & 1.879 & 0.085 \\
\hline & II & $\begin{array}{c}6.262 \\
\pm \\
0.644\end{array}$ & $\begin{array}{c}6.730 \\
\pm \\
0.709\end{array}$ & $\begin{array}{c}7.136 \\
\pm \\
0.345\end{array}$ & $\begin{array}{c}5.742 \\
\pm \\
0.681\end{array}$ & $\begin{array}{c}6.468 \\
\pm \\
0.775\end{array}$ & & & & \\
\hline & III & $\begin{array}{c}6.420 \\
\pm \\
1.497\end{array}$ & $\begin{array}{c}7.682 \\
\pm \\
1.592\end{array}$ & $\begin{array}{c}7.794 \\
\pm \\
0.360\end{array}$ & $\begin{array}{c}6.042 \\
\pm \\
0.244\end{array}$ & $\begin{array}{c}6.985 \\
\pm \\
1.290\end{array}$ & Site & 24 & 0.373 & 0.997 \\
\hline & IV & $\begin{array}{c}7.774 \\
\pm \\
1.037\end{array}$ & $\begin{array}{c}8.138 \\
\pm \\
0.755\end{array}$ & $\begin{array}{c}8.286 \\
\pm \\
0.314\end{array}$ & $\begin{array}{c}7.886 \\
\pm \\
0.747\end{array}$ & $\begin{array}{c}8.021 \\
\pm \\
0.726\end{array}$ & $\mathrm{x}$ & & & \\
\hline & V & $\begin{array}{c}8.014 \\
\pm \\
0.872\end{array}$ & $\begin{array}{c}8.522 \\
\pm \\
0.476\end{array}$ & $\begin{array}{c}9.554 \\
\pm \\
1.388\end{array}$ & $\begin{array}{c}8.296 \\
\pm \\
0.232\end{array}$ & $\begin{array}{c}8.597 \\
\pm \\
0.990\end{array}$ & Season & & & \\
\hline \multirow{5}{*}{ Muscles } & I & $\begin{array}{c}3.286 \\
\pm \\
0.303\end{array}$ & $\begin{array}{c}3.912 \\
\pm \\
0.719\end{array}$ & $\begin{array}{c}4.682 \\
\pm \\
0.914\end{array}$ & $\begin{array}{c}3.790 \\
\pm \\
0.542\end{array}$ & $\begin{array}{c}3.918 \\
\pm \\
0.793\end{array}$ & $\mathrm{x}$ & & & \\
\hline & II & $\begin{array}{c}3.432 \\
\pm \\
0.668\end{array}$ & $\begin{array}{c}3.550 \\
\pm \\
0.382\end{array}$ & $\begin{array}{c}3.834 \\
\pm \\
0.926\end{array}$ & $\begin{array}{c}3.030 \\
\pm \\
0.268\end{array}$ & $\begin{array}{c}3.462 \\
\pm \\
0.639\end{array}$ & Organ & & & \\
\hline & III & $\begin{array}{c}3.332 \\
\pm \\
0.200\end{array}$ & $\begin{array}{c}3.810 \\
\pm \\
1.143\end{array}$ & $\begin{array}{c}4.124 \\
\pm \\
0.158\end{array}$ & $\begin{array}{c}3.776 \\
\pm \\
0.468\end{array}$ & $\begin{array}{c}3.761 \\
\pm \\
0.647\end{array}$ & & & & \\
\hline & IV & $\begin{array}{c}3.834 \\
\pm \\
0.225\end{array}$ & $\begin{array}{c}4.294 \\
\pm \\
1.061\end{array}$ & $\begin{array}{c}4.890 \\
\pm \\
0.713\end{array}$ & $\begin{array}{c}4.042 \\
\pm \\
0.290\end{array}$ & $\begin{array}{c}4.265 \\
\pm \\
0.737\end{array}$ & & & & \\
\hline & V & $\begin{array}{c}3.736 \\
\pm \\
0.443\end{array}$ & $\begin{array}{c}4.660 \\
\pm \\
0.203\end{array}$ & $\begin{array}{c}5.466 \\
\pm \\
0.359\end{array}$ & $\begin{array}{c}4.354 \\
\pm \\
0.188\end{array}$ & $\begin{array}{c}4.554 \\
\pm \\
0.702\end{array}$ & & & & \\
\hline
\end{tabular}


Table. 6: Seasonal variations of Zinc concentration ( $\mu \mathrm{g} / \mathrm{g}$ dry weight) in different organs of Liza ramada from Lake Manzala .

\begin{tabular}{|c|c|c|c|c|c|c|c|c|c|c|}
\hline \multirow{2}{*}{ Organ } & \multirow{2}{*}{ Site } & \multicolumn{5}{|c|}{ Seasons } & \multicolumn{4}{|c|}{ ANOVA } \\
\hline & & Winter & Spring & Summer & Autumn & Total & Factor & $\mathrm{df}$ & $F$ value & Sig. \\
\hline \multirow{5}{*}{ Gills } & I & $\begin{array}{c}55.100 \\
\pm \\
2.191\end{array}$ & $\begin{array}{c}82.100 \\
\pm \\
1.543\end{array}$ & $\begin{array}{c}87.600 \\
\pm \\
2.929\end{array}$ & $\begin{array}{c}49.800 \\
\pm \\
5.635\end{array}$ & $\begin{array}{c}68.650 \\
\pm \\
17.128\end{array}$ & Site & 4 & $\begin{array}{c}1143.3 \\
04\end{array}$ & $\begin{array}{l}0.000 * \\
* *\end{array}$ \\
\hline & II & $\begin{array}{c}46.900 \\
\pm \\
2.977\end{array}$ & $\begin{array}{c}51.020 \\
\pm \\
2.560\end{array}$ & $\begin{array}{c}70.800 \\
\pm \\
3.555\end{array}$ & $\begin{array}{c}48.500 \\
\pm \\
2.239\end{array}$ & $\begin{array}{c}54.310 \\
\pm \\
10.241\end{array}$ & Season & 3 & $\begin{array}{c}1718.5 \\
86\end{array}$ & $\begin{array}{l}0.000 * \\
* *\end{array}$ \\
\hline & III & $\begin{array}{c}47.600 \\
\pm \\
1.433\end{array}$ & $\begin{array}{c}61.300 \\
\pm \\
2.971\end{array}$ & $\begin{array}{c}81.400 \\
\pm \\
3.488\end{array}$ & $\begin{array}{c}66.100 \\
\pm \\
1.834\end{array}$ & $\begin{array}{c}64.100 \\
\pm \\
12.611\end{array}$ & Organ & 2 & $\begin{array}{l}9394.5 \\
75\end{array}$ & $\begin{array}{l}0.000^{*} \\
* *\end{array}$ \\
\hline & IV & $\begin{array}{c}60.100 \\
\pm \\
2.599\end{array}$ & $\begin{array}{c}89.400 \\
\pm \\
2.660\end{array}$ & $\begin{array}{c}98.600 \\
\pm \\
2.907\end{array}$ & $\begin{array}{c}75.700 \\
\pm \\
3.421\end{array}$ & $\begin{array}{c}80.950 \\
\pm \\
15.161\end{array}$ & $\begin{array}{c}\text { Site } \mathrm{x} \\
\text { Season }\end{array}$ & 12 & 52.240 & $\begin{array}{l}0.000 * \\
* *\end{array}$ \\
\hline & $\mathrm{V}$ & $\begin{array}{c}65.700 \\
\pm \\
3.806\end{array}$ & $\begin{array}{c}112.600 \\
\pm \\
2.732\end{array}$ & $\begin{array}{c}138.300 \\
\pm \\
3.265\end{array}$ & $\begin{array}{c}97.800 \\
\pm \\
1.425\end{array}$ & $\begin{array}{c}103.600 \\
\pm \\
27.056\end{array}$ & $\begin{array}{l}\text { Site } x \\
\text { Organ }\end{array}$ & 8 & $\begin{array}{c}127.76 \\
5\end{array}$ & $\begin{array}{l}0.000 * \\
* *\end{array}$ \\
\hline \multirow{5}{*}{ Skin } & I & $\begin{array}{c}31.100 \\
\pm \\
1.598\end{array}$ & $\begin{array}{c}52.800 \\
\pm \\
1.557\end{array}$ & $\begin{array}{c}69.800 \\
\pm \\
2.253\end{array}$ & $\begin{array}{c}33.400 \\
\pm \\
2.236\end{array}$ & $\begin{array}{c}46.780 \\
\pm \\
16.279\end{array}$ & $\begin{array}{c}\text { Season x } \\
\text { Organ }\end{array}$ & 6 & $\begin{array}{c}243.34 \\
7\end{array}$ & $\begin{array}{l}0.000 * \\
* *\end{array}$ \\
\hline & II & $\begin{array}{c}27.100 \\
\pm \\
1.548\end{array}$ & $\begin{array}{c}35.900 \\
\pm \\
5.436\end{array}$ & $\begin{array}{c}53.100 \\
\pm \\
2.281\end{array}$ & $\begin{array}{c}28.100 \\
\pm \\
2.504\end{array}$ & $\begin{array}{c}35.900 \\
\pm \\
10.867\end{array}$ & & & & \\
\hline & III & $\begin{array}{c}29.400 \\
\pm \\
2.535\end{array}$ & $\begin{array}{c}51.400 \\
\pm \\
2.174\end{array}$ & $\begin{array}{c}64.300 \\
\pm \\
2.574\end{array}$ & $\begin{array}{c}30.800 \\
\pm \\
2.482\end{array}$ & $\begin{array}{c}43.980 \\
\pm \\
15.161\end{array}$ & Site & 24 & 16.990 & $\begin{array}{l}0.000 * \\
* *\end{array}$ \\
\hline & IV & $\begin{array}{c}32.600 \\
\pm \\
1.803\end{array}$ & $\begin{array}{c}61.100 \\
\pm \\
2.560\end{array}$ & $\begin{array}{c}77.500 \\
\pm \\
3.320\end{array}$ & $\begin{array}{c}41.100 \\
\pm \\
2.527\end{array}$ & $\begin{array}{c}53.080 \\
\pm \\
18.112\end{array}$ & $\mathrm{x}$ & & & \\
\hline & $\mathrm{V}$ & $\begin{array}{c}34.700 \\
\pm \\
2.603\end{array}$ & $\begin{array}{c}86.400 \\
\pm \\
3.690\end{array}$ & $\begin{array}{c}75.800 \\
\pm \\
3.406\end{array}$ & $\begin{array}{c}55.300 \\
\pm \\
3.958\end{array}$ & $\begin{array}{c}63.050 \\
\pm \\
20.572\end{array}$ & Season & & & \\
\hline \multirow{5}{*}{ Muscles } & I & $\begin{array}{c}18.400 \\
\pm \\
3.362\end{array}$ & $\begin{array}{c}22.400 \\
\pm \\
2.527\end{array}$ & $\begin{array}{c}25.100 \\
\pm \\
3.175\end{array}$ & $\begin{array}{c}20.400 \\
\pm \\
3.130\end{array}$ & $\begin{array}{c}21.575 \\
\pm \\
3.793\end{array}$ & $\mathrm{x}$ & & & \\
\hline & II & $\begin{array}{c}12.600 \\
\pm \\
1.565\end{array}$ & $\begin{array}{c}16.100 \\
\pm \\
1.610\end{array}$ & $\begin{array}{c}19.200 \\
\pm \\
2.303\end{array}$ & $\begin{array}{c}14.600 \\
\pm \\
1.543\end{array}$ & $\begin{array}{c}15.625 \\
\pm \\
2.963\end{array}$ & Organ & & & \\
\hline & III & $\begin{array}{c}16.500 \\
\pm \\
2.460\end{array}$ & $\begin{array}{c}19.700 \\
\pm \\
2.661\end{array}$ & $\begin{array}{c}18.400 \\
\pm \\
2.460\end{array}$ & $\begin{array}{c}21.400 \\
\pm \\
2.351\end{array}$ & $\begin{array}{c}19.000 \\
\pm \\
2.928\end{array}$ & & & & \\
\hline & IV & $\begin{array}{c}22.200 \\
\pm \\
2.422\end{array}$ & $\begin{array}{c}26.800 \\
\pm \\
3.555\end{array}$ & $\begin{array}{c}31.010 \\
\pm \\
2.415\end{array}$ & $\begin{array}{c}23.700 \\
\pm \\
2.411\end{array}$ & $\begin{array}{c}25.930 \\
\pm \\
4.293\end{array}$ & & & & \\
\hline & $\mathrm{V}$ & $\begin{array}{c}28.900 \\
\pm \\
1.945\end{array}$ & $\begin{array}{c}32.900 \\
\pm \\
1.453\end{array}$ & $\begin{array}{c}36.900 \\
\pm \\
2.418\end{array}$ & $\begin{array}{c}24.300 \\
\pm \\
1.476\end{array}$ & $\begin{array}{c}30.750 \\
\pm \\
5.093\end{array}$ & & & & \\
\hline
\end{tabular}


Table. 7: Seasonal variations of cadmium concentration ( $\mu \mathrm{g} / \mathrm{g}$ dry weight) in different organs of Liza ramada from Lake Manzala .

\begin{tabular}{|c|c|c|c|c|c|c|c|c|c|c|}
\hline \multirow{2}{*}{ Organ } & \multirow{2}{*}{ Site } & \multicolumn{5}{|c|}{ Seasons } & \multicolumn{4}{|c|}{ ANOVA } \\
\hline & & Winter & Spring & Summer & Autumn & Total & Factor & $\mathrm{df}$ & $\mathrm{F}$ value & Sig. \\
\hline \multirow{5}{*}{ Gills } & I & $\begin{array}{c}2.346 \\
\pm \\
0.585\end{array}$ & $\begin{array}{c}3.684 \\
\pm \\
0.453\end{array}$ & $\begin{array}{c}4.220 \\
\pm \\
0.470 \\
\end{array}$ & $\begin{array}{c}2.820 \\
\pm \\
0.701\end{array}$ & $\begin{array}{c}3.270 \\
\pm \\
0.854\end{array}$ & Site & 4 & 76.491 & $\underset{* *}{0.000 *}$ \\
\hline & II & $\begin{array}{c}1.930 \\
\pm \\
1.185\end{array}$ & $\begin{array}{c}2.364 \\
\pm \\
0.546\end{array}$ & $\begin{array}{c}3.200 \\
\pm \\
0.313 \\
\end{array}$ & $\begin{array}{c}1.630 \\
\pm \\
0.516\end{array}$ & $\begin{array}{c}2.280 \\
\pm \\
0.890 \\
\end{array}$ & Season & 3 & 41.432 & $* *^{0.000^{*}}$ \\
\hline & III & $\begin{array}{c}1.888 \\
\pm \\
0.760\end{array}$ & $\begin{array}{c}2.618 \\
\quad \pm \\
1.180\end{array}$ & $\begin{array}{c}4.170 \\
\pm \\
0.604 \\
\end{array}$ & $\begin{array}{c}1.908 \\
\pm \\
0.263\end{array}$ & $\begin{array}{c}2.650 \\
\quad \pm \\
1.207 \\
\end{array}$ & Organ & 2 & 984.556 & ${ }_{* *}^{0.000 *}$ \\
\hline & IV & $\begin{array}{c}3.278 \\
\pm \\
0.883\end{array}$ & $\begin{array}{c}4.088 \\
\pm \\
0.830\end{array}$ & $\begin{array}{c}5.040 \\
\pm \\
0.962 \\
\end{array}$ & $\begin{array}{c}3.780 \\
\pm \\
0.325 \\
\end{array}$ & $\begin{array}{c}4.050 \\
\pm \\
0.884 \\
\end{array}$ & $\begin{array}{c}\text { Site x } \\
\text { Season }\end{array}$ & 12 & 0.384 & 0.968 \\
\hline & V & $\begin{array}{c}2.274 \\
\pm \\
1.528\end{array}$ & $\begin{array}{c}5.392 \\
\pm \\
0.617\end{array}$ & $\begin{array}{c}5.920 \\
\pm \\
0.872\end{array}$ & $\begin{array}{c}4.852 \\
\pm \\
0.800\end{array}$ & $\begin{array}{c}5.160 \\
\pm \\
1.073\end{array}$ & $\begin{array}{l}\text { Site } \mathrm{x} \\
\text { Organ }\end{array}$ & 8 & 28.377 & ${ }_{* *}^{0.000 *}$ \\
\hline \multirow{5}{*}{ Skin } & I & $\begin{array}{c}1.170 \\
\pm \\
0.051\end{array}$ & $\begin{array}{c}1.120 \\
\pm \\
0.123\end{array}$ & $\begin{array}{c}1.410 \\
\pm \\
0.111 \\
\end{array}$ & $\begin{array}{c}1.235 \\
\pm \\
0.071\end{array}$ & $\begin{array}{c}1.240 \\
\quad \pm \\
0.148 \\
\end{array}$ & $\begin{array}{c}\text { Season x } \\
\text { Organ }\end{array}$ & 6 & 16.514 & ${ }_{* *}^{0.000 *}$ \\
\hline & II & $\begin{array}{c}1.038 \\
\pm \\
0.103\end{array}$ & $\begin{array}{c}1.086 \\
\pm \\
0.069\end{array}$ & $\begin{array}{c}1.180 \\
\pm \\
0.042 \\
\end{array}$ & $\begin{array}{c}1.109 \\
\pm \\
0.046\end{array}$ & $\begin{array}{c}1.132 \\
\pm \\
0.182 \\
\end{array}$ & & & & \\
\hline & III & $\begin{array}{c}1.110 \\
\pm \\
0.042\end{array}$ & $\begin{array}{c}1.020 \\
\pm \\
0.069\end{array}$ & $\begin{array}{c}1.206 \\
\pm \\
0.148\end{array}$ & $\begin{array}{c}1.127 \\
\pm \\
0.057\end{array}$ & $\begin{array}{c}1.172 \\
\pm \\
0.188\end{array}$ & Site & 24 & 0.613 & 0.923 \\
\hline & IV & $\begin{array}{c}1.468 \\
\pm \\
0.103\end{array}$ & $\begin{array}{c}1.268 \\
\pm \\
0.092\end{array}$ & $\begin{array}{c}1.782 \\
\pm \\
0.066 \\
\end{array}$ & $\begin{array}{c}1.512 \\
\quad \pm \\
0.124\end{array}$ & $\begin{array}{c}1.530 \\
\pm \\
0.633 \\
\end{array}$ & $\mathrm{x}$ & & & \\
\hline & V & $\begin{array}{c}1.680 \\
\pm \\
0.246\end{array}$ & $\begin{array}{c}1.570 \\
\pm \\
0.410\end{array}$ & $\begin{array}{c}\begin{array}{c}2.146 \\
\pm \\
0.115\end{array} \\
\end{array}$ & $\begin{array}{c}1.837 \\
\pm \\
0.173\end{array}$ & $\begin{array}{c}1.952 \\
\pm \\
0.530\end{array}$ & Season & & & \\
\hline \multirow{5}{*}{ Muscles } & I & $\begin{array}{c}0.566 \\
\pm \\
0.091\end{array}$ & $\begin{array}{c}0.660 \\
\pm \\
0.022\end{array}$ & $\begin{array}{c}0.780 \\
\pm \\
0.074 \\
\end{array}$ & $\begin{array}{c}0.600 \\
\pm \\
0.039\end{array}$ & $\begin{array}{c}0.650 \\
\pm \\
0.107\end{array}$ & $\mathrm{x}$ & & & \\
\hline & II & $\begin{array}{c}0.508 \\
\pm \\
0.078\end{array}$ & $\begin{array}{c}0.570 \\
\pm \\
0.058\end{array}$ & $\begin{array}{c}0.622 \\
\pm \\
0.072\end{array}$ & $\begin{array}{c}0.560 \\
\pm \\
0.082\end{array}$ & $\begin{array}{c}0.570 \\
\pm \\
0.089\end{array}$ & Organ & & & \\
\hline & III & $\begin{array}{c}0.560 \\
\pm \\
0.066\end{array}$ & $\begin{array}{c}0.620 \\
\pm \\
0.067\end{array}$ & $\begin{array}{c}0.652 \\
\pm \\
0.070\end{array}$ & $\begin{array}{c}0.580 \\
\pm \\
0.070\end{array}$ & $\begin{array}{c}0.604 \\
\pm \\
0.073\end{array}$ & & & & \\
\hline & IV & $\begin{array}{c}0.588 \\
\pm \\
0.145\end{array}$ & $\begin{array}{c}0.810 \\
\pm \\
0.066\end{array}$ & $\begin{array}{c}0.920 \\
\pm \\
0.089\end{array}$ & $\begin{array}{c}0.620 \\
\pm \\
0.067\end{array}$ & $\begin{array}{c}0.740 \\
\pm \\
0.179\end{array}$ & & & & \\
\hline & $\mathrm{V}$ & $\begin{array}{c}0.634 \\
\pm \\
0.036\end{array}$ & $\begin{array}{c}0.970 \\
\pm \\
0.086\end{array}$ & $\begin{array}{c}1.112 \\
\pm \\
0.155\end{array}$ & $\begin{array}{c}0.690 \\
\pm \\
0.087\end{array}$ & $\begin{array}{c}0.853 \\
\pm \\
0.221\end{array}$ & & & & \\
\hline
\end{tabular}


Table. 8: Seasonal variations of lead concentration ( $\mu \mathrm{g} / \mathrm{g}$ dry weight) in different organs of Liza ramada from Lake Manzala .

\begin{tabular}{|c|c|c|c|c|c|c|c|c|c|c|}
\hline \multirow{2}{*}{ Organ } & \multirow{2}{*}{ Site } & \multicolumn{5}{|c|}{ Seasons } & \multicolumn{4}{|c|}{ ANOVA } \\
\hline & & Winter & Spring & Summer & Autumn & Total & Factor & $\mathrm{df}$ & F value & Sig. \\
\hline \multirow{5}{*}{ Gills } & I & $\begin{array}{c}7.956 \\
\pm \\
0.683\end{array}$ & $\begin{array}{c}8.620 \\
\pm \\
0.268\end{array}$ & $\begin{array}{c}8.846 \\
\pm \\
1252\end{array}$ & $\begin{array}{c}8.336 \\
\pm \\
0.69\end{array}$ & $\begin{array}{c}8.440 \\
\pm \\
0831\end{array}$ & Site & 4 & 31.690 & $0.000 * * *$ \\
\hline & II & $\begin{array}{c}5.364 \\
\pm \\
1.181\end{array}$ & $\begin{array}{c}5.580 \\
\pm \\
0.537\end{array}$ & $\begin{array}{c}5.774 \\
\pm \\
0.795\end{array}$ & $\begin{array}{c}5.508 \\
\pm \\
1.006\end{array}$ & $\begin{array}{c}5.560 \\
\pm \\
0.850\end{array}$ & Season & 3 & 3.084 & $0.028^{*}$ \\
\hline & III & $\begin{array}{c}6.316 \\
\pm \\
1.033\end{array}$ & $\begin{array}{c}6.820 \\
\pm \\
0.581\end{array}$ & $\begin{array}{c}7.050 \\
\pm \\
0.829\end{array}$ & $\begin{array}{c}6.656 \\
\pm \\
0.835\end{array}$ & $\begin{array}{c}6.710 \\
\pm \\
0.805\end{array}$ & Organ & 2 & $\begin{array}{c}734.96 \\
8\end{array}$ & $0.000 * * *$ \\
\hline & IV & $\begin{array}{c}8.326 \\
\pm \\
0.813\end{array}$ & $\begin{array}{c}9.370 \\
\pm \\
1.811\end{array}$ & $\begin{array}{c}9.942 \\
\pm \\
2.890\end{array}$ & $\begin{array}{c}9.116 \\
\pm \\
0.757\end{array}$ & $\begin{array}{c}9.190 \\
\pm \\
1.744\end{array}$ & $\begin{array}{l}\text { Site } \mathrm{x} \\
\text { Season }\end{array}$ & 12 & 0.630 & 0.816 \\
\hline & $\mathrm{V}$ & $\begin{array}{c}9.224 \\
\pm \\
0.825\end{array}$ & $\begin{array}{c}10.010 \\
\pm \\
1.319\end{array}$ & $\begin{array}{c}11.524 \\
\pm \\
2.317 \\
\end{array}$ & $\begin{array}{c}9.776 \\
\pm \\
1.492 \\
\end{array}$ & $\begin{array}{c}10.130 \\
\pm \\
1.699\end{array}$ & $\begin{array}{l}\text { Site } \mathrm{x} \\
\text { Organ }\end{array}$ & 8 & 10.629 & $0.000 * * *$ \\
\hline \multirow{5}{*}{ Skin } & I & $\begin{array}{c}2.540 \\
\pm \\
0.431\end{array}$ & $\begin{array}{c}2.676 \\
\pm \\
0.083\end{array}$ & $\begin{array}{c}2.744 \\
\pm \\
0.314\end{array}$ & $\begin{array}{c}2.660 \\
\pm \\
0.537\end{array}$ & $\begin{array}{c}2.676 \\
\pm \\
0.166\end{array}$ & $\begin{array}{c}\text { Season } \mathrm{x} \\
\text { Organ }\end{array}$ & 6 & 1.486 & 0.184 \\
\hline & II & $\begin{array}{c}2.420 \\
\pm \\
0.447\end{array}$ & $\begin{array}{c}2.426 \\
\pm \\
0.277\end{array}$ & $\begin{array}{c}2.522 \\
\pm \\
0.146\end{array}$ & $\begin{array}{c}2.310 \\
\pm \\
0.488\end{array}$ & $\begin{array}{c}2.420 \\
\pm \\
0.358\end{array}$ & & & & \\
\hline & III & $\begin{array}{c}2.410 \\
\pm \\
0.472\end{array}$ & $\begin{array}{c}2.544 \\
\pm \\
0.539\end{array}$ & $\begin{array}{c}2.630 \\
\pm \\
0.082\end{array}$ & $\begin{array}{c}2.490 \\
\pm \\
0.447\end{array}$ & $\begin{array}{c}2.520 \\
\pm \\
0.402\end{array}$ & Site & 24 & 0.805 & 0.73 \\
\hline & IV & $\begin{array}{c}2.686 \\
\pm \\
0.095\end{array}$ & $\begin{array}{c}2.872 \\
\pm \\
0.149\end{array}$ & $\begin{array}{c}2.962 \\
\pm \\
0.281\end{array}$ & $\begin{array}{c}2.730 \\
\pm \\
0.157\end{array}$ & $\begin{array}{c}2.810 \\
\pm \\
0.224\end{array}$ & $\mathrm{x}$ & & & \\
\hline & $\mathrm{V}$ & $\begin{array}{c}2.776 \\
\pm \\
0.083\end{array}$ & $\begin{array}{c}3.308 \\
\pm \\
0.493\end{array}$ & $\begin{array}{c}3.110 \\
\pm \\
0.294\end{array}$ & $\begin{array}{c}2.960 \\
\pm \\
0.134 \\
\end{array}$ & $\begin{array}{c}3.040 \\
\pm \\
0.358\end{array}$ & Season & & & \\
\hline \multirow{5}{*}{ Muscles } & I & $\begin{array}{c}1.532 \\
\pm \\
0.259\end{array}$ & $\begin{array}{c}1.660 \\
\pm \\
0.248\end{array}$ & $\begin{array}{c}1.830 \\
\pm \\
0.216\end{array}$ & $\begin{array}{c}1.612 \\
\pm \\
0.480\end{array}$ & $\begin{array}{c}1.659 \\
\pm \\
0.318\end{array}$ & $\mathrm{x}$ & & & \\
\hline & II & $\begin{array}{c}1.432 \\
\pm \\
0.159\end{array}$ & $\begin{array}{c}1.540 \\
\pm \\
0.134\end{array}$ & $\begin{array}{c}1.650 \\
\pm \\
0.201\end{array}$ & $\begin{array}{c}1.472 \\
\pm \\
0.140\end{array}$ & $\begin{array}{c}1.526 \\
\pm \\
0.169\end{array}$ & Organ & & & \\
\hline & III & $\begin{array}{c}1.462 \\
\pm \\
0.211\end{array}$ & $\begin{array}{c}1.630 \\
\pm \\
0.224\end{array}$ & $\begin{array}{c}1.700 \\
\pm \\
0.243\end{array}$ & $\begin{array}{c}1.518 \\
\pm \\
0.072\end{array}$ & $\begin{array}{c}1.576 \\
\pm \\
0.202\end{array}$ & & & & \\
\hline & IV & $\begin{array}{c}1.994 \\
\pm \\
0.303\end{array}$ & $\begin{array}{c}2.070 \\
\pm \\
0.112\end{array}$ & $\begin{array}{c}2.240 \\
\pm \\
0.402\end{array}$ & $\begin{array}{c}2.044 \\
\pm \\
0.149\end{array}$ & $\begin{array}{c}2.087 \\
\pm \\
0.267\end{array}$ & & & & \\
\hline & V & $\begin{array}{c}2.060 \\
\pm \\
0.352\end{array}$ & $\begin{array}{c}2.430 \\
\pm \\
0.313\end{array}$ & $\begin{array}{c}2.230 \\
\pm \\
0.291\end{array}$ & $\begin{array}{c}2.120 \\
\pm \\
0.730\end{array}$ & $\begin{array}{c}2.210 \\
\pm \\
0.365\end{array}$ & & & & \\
\hline
\end{tabular}

